\title{
Multiphase modeling to predict finite deformations of elastic objects in free surface flows
}

\author{
S. Ushijima ${ }^{1} \&$ N. Kuroda ${ }^{2}$ \\ ${ }^{1}$ Kyoto University, ACCMS, Kyoto 606-8501, Japan \\ ${ }^{2}$ Kyoto University, CERE, Kyoto 615-8540, Japan
}

\begin{abstract}
This paper deals with a computational method based on multiphase modeling to predict the interactions between free-surface flows and linear elastic objects that undergo finite deformations. In multiphase modeling, a field consisting of gas, liquid and solid phases is treated as the immiscible and incompressible fluid-mixture with different physical properties and the governing equations are derived with one-fluid modeling, while the solid model is derived with objective stress rates and discretization in FEM to deal with the finite deformations. Applying the proposed computational method to experimental results, it was shown that the time histories of the displacements of an elastic plate and the fluid forces caused by wave-induced flows are successfully predicted and that the differences of the fluid forces acting on elastic and rigid plates are reasonably predicted.

Keywords: fluid-solid interaction, free-surface flow, elastic body, finite deformation, multiphase model.
\end{abstract}

\section{Introduction}

The accurate evaluation of the interactions between free-surface flows and the deformations of elastic objects is an important engineering subject, as found in the dynamic responses of floating elastic structures against wave motions and the fluid resistance forces of the flexible plants in river flows. While many investigations have been made for one and two-degree of freedom problems such as the oscillations of a cylinder in uniform flows, the numerical studies for multi-degree of freedom are relatively few, in particular for the interactions between free surface flows and finite deformations of objects. 
In the present study, a flexible object is represented by multiple tetrahedron elements and the finite deformations are calculated with stress rates and a finite element method (FEM). This solid model is implemented in a multiphase model to take account of the fluid-solid interaction. In the multiphase model, the freesurface flow including solid objects, which consists of gas, liquid and solid phases, is treated as a multiphase field and it is modeled as a mixture of the immiscible and incompressible different fluids. The fluid-solid interactions are dealt with the fluid forces, which are calculated from the momentum equation of the multiphase model, and mass-averaged velocities evaluated with the object displacement velocities.

The computational method was applied to the experiments, in which the finite deformations of an elastic plate and the fluid forces were measured in a water tank equipped with a wave generator. As a result, it was shown that the time histories of the plate displacements and fluid forces are successfully predicted with the present method. In addition, it was demonstrated that the differences of the fluid forces acting on elastic and rigid plates are reasonably predicted.

\section{Numerical procedures}

\subsection{Multiphase modeling}

It is necessary to evaluate fluid forces acting on solid objects accurately in the prediction of fluid-solid interactions. The fluid forces are usually obtained through the surface integral of the fluid pressure and viscous stresses around the object with boundary-fitted coordinates or unstructured elements. However, this approach becomes difficult when the object shapes are largely complicated and they collide with each other.

In order to propose more robust computational methods, multiphase modeling [1], [2] is employed in this study; a field consisting of gas, liquid and solid phases is assumed to be immiscible and incompressible fluid-mixture with the physical properties corresponding to their phases. The fluid forces acting on the solid objects are evaluated with the volume integral of the pressure and viscous terms in the momentum equations for multiphase flows. The governing equations are the mass conservation equation in Eulerian description, incompressible condition and momentum equation in conservation form:

$$
\begin{gathered}
\frac{\partial \rho}{\partial t}+\frac{\partial}{\partial x_{j}}\left(\rho u_{j}\right)=0 \\
\frac{\partial u_{j}}{\partial x_{j}}=0 \\
\frac{\partial u_{i}}{\partial t}+\frac{\partial}{\partial x_{j}}\left(u_{i} u_{j}\right)=f_{i}-\frac{1}{\rho} \frac{\partial p}{\partial x_{i}}+\frac{1}{\rho} \frac{\partial}{\partial x_{j}}\left[\frac{\partial}{\partial x_{j}}\left(\mu u_{i}\right)+\frac{\partial}{\partial x_{i}}\left(\mu u_{j}\right)\right]
\end{gathered}
$$

where $t$ is time, $x_{i}$ is the component of three-dimensional orthogonal coordinates and $f_{i}$ is the acceleration component of the external forces. While the velocity 
component $u_{i}$ is the mass-averaged value in the mixture of fluids, the density $\rho$, pressure $p$ and viscous coefficient $\mu$ are defined as the volume-averaged values. The basic equations are discretized in a collocated grid system [3] on the basis of a finite volume method. The numerical procedures to solve the basic equations are based on a MAC method [4], in which some improved numerical techniques are implemented, such as an implicit solver (C-ISMAC method) [5] and a pressure computation method (C-HSMAC method) [6] effective to the incompressible fluids [7].

\subsection{Solid model}

The movement of a deformable body $\Omega$ is described with the principle of virtual work as follows:

$$
\int_{\Omega} \boldsymbol{v}^{* T} \rho \ddot{\boldsymbol{v}} d \Omega+\int_{\Omega} \boldsymbol{v}^{* T} c \dot{\boldsymbol{v}} d \Omega+\int_{\Omega} \boldsymbol{\epsilon}^{* T} \boldsymbol{\sigma} d \Omega=\int_{\Omega} \boldsymbol{v}^{* T} \boldsymbol{f}_{e} d \Omega
$$

where $\rho, c, \boldsymbol{v}, \boldsymbol{\sigma}$ and $\boldsymbol{f}_{e}$ are density, damping coefficient, displacement, stress and external force vectors, respectively. The virtual displacement and strain vectors are defined as $\boldsymbol{v}^{*}$ and $\boldsymbol{\epsilon}^{*}$. The dots on the variables indicate the time derivatives. The solid object is represented with the second-order tetrahedron elements used in FEM, in which each element has ten nodes. The shape function $N_{i}(i=1, \cdots, 10)$ in the element is given by a quadratic function of the natural coordinates in the corresponding isoparametric element. When $\boldsymbol{v}$ and $\boldsymbol{v}^{*}$ are discretized with $N_{i}$, (4) can be rewritten with the variables defined on the nodes.

$$
M \ddot{\boldsymbol{d}}+C \dot{\boldsymbol{d}}+\boldsymbol{F}_{\text {int }}=\boldsymbol{f}
$$

where $\boldsymbol{d}$ and $\boldsymbol{f}$ are the node displacement vector and the external force vector acting on the nodes, while $M$ and $C$ are mass and damping matrices, which are given by diagonal forms.

A solid object treated in this study is assumed to be a linear elastic body. Thus, the stress strain behavior of the material is given by a linear constitutive relation, while the finite deformation, which is related to the geometric nonlinearity [8], is taken into account. In order to deal with the large deformation, the following objective stress rate, Cotter-Rivlin stress rate tensor $\dot{T}_{c}$, is utilized [9]:

$$
\dot{T}_{c}=\dot{T}+L^{T} T+T L
$$

where $T$ and $L$ are Cauchy stress and velocity gradient tensors, respectively. The $\dot{T}_{c}$ is evaluated with the following time derivative of the linear constitutive relation:

$$
\left\{\dot{T}_{c}\right\}_{v}=D \dot{\boldsymbol{\epsilon}}=D B \dot{\boldsymbol{d}}
$$

where \{\}$_{v}$ means the vector form of the tensor, $\epsilon$ is the strain vector on nodes, and $D$ and $B$ are stress-strain and strain-displacement matrices. The stress tensor at $n+$ 
1 time step given by $T^{n+1}$ is approximated with the Euler explicit discretization:

$$
\left\{T^{n+1}\right\}_{v}=\left\{T^{n}\right\}_{v}+\{\dot{T}\}_{v} \Delta t
$$

where $\dot{T}$ is obtained from eqn. (6). Then, $\boldsymbol{F}_{i n t}^{n+1}$ in eqn. (5) is calculated with the following volume integral:

$$
\boldsymbol{F}_{i n t}^{n+1}=\int_{\Omega} \boldsymbol{B}^{T}\left\{T^{n+1}\right\}_{v} d \Omega
$$

Finally, the acceleration vector $\ddot{d}$ is calculated from eqn. (5) with the following equation:

$$
\ddot{d}=M^{-1}\left[f-C \dot{d}-F_{i n t}\right]
$$

The displacement vector $\boldsymbol{d}$ can be obtained by the numerical time integration of eqn. (10).

\subsection{Interaction between fluids and solid objects}

The fluid forces acting on the object are determined from the pressure and viscous terms of eqn. (3). In the solid model described above, the fluid forces at the nodes need to be calculated. Thus, the fluid force is firstly estimated for a part of the tetrahedron element included in a fluid-cell, as illustrated in Fig. 1 and then it is distributed to the nodes. It is noted that Fig. 1 is schematically shown with a firstorder element that has four nodes.

In Fig. 1, $\boldsymbol{F}_{C k m}$ indicates the fluid force vector acting on a part of the element $T_{k m}$ of the object- $k$ included in fluid-cell $C$. The $x_{i}$ component of $\boldsymbol{F}_{C k m}$, which is given by $F_{C k m}^{i}$, is calculated with a portion of the element volume $\Delta T_{C k m}$ included in the fluid-cell $C$ and the density $\rho_{b k}$ of the object $-k$ as

$$
F_{C k m}^{i}=\rho_{b k} \Delta T_{C k m}\left[-\frac{1}{\rho} \frac{\partial p}{\partial x_{i}}+\frac{1}{\rho} \frac{\partial}{\partial x_{j}}\left\{\frac{\partial}{\partial x_{j}}\left(\mu u_{i}\right)+\frac{\partial}{\partial x_{i}}\left(\mu u_{j}\right)\right\}\right]
$$

where $\Delta T_{C k m}$ is estimated with the sub-cell method [7]. The fluid force vector $\boldsymbol{F}_{C k m}$ calculated from eqn. (11) is distributed to the element nodes.

On the other hand, the response of the dynamic behaviors of the object is taken into account in the multiphase field, as schematically shown in Fig. 2.

The velocity vector $\boldsymbol{v}_{\mathrm{km}}$ of the tetrahedron element is determined as the average value of those defined at the nodes $\dot{\boldsymbol{d}}$, which is equivalent to the node velocity vector $\boldsymbol{v}_{k m j}$ in Fig. 2. The contribution of the element of $T_{k m}$ to the fluid-cell $C$ is then determined with $\boldsymbol{v}_{k m}$, density and volume $\Delta T_{C k m}$. Finally, the velocity 


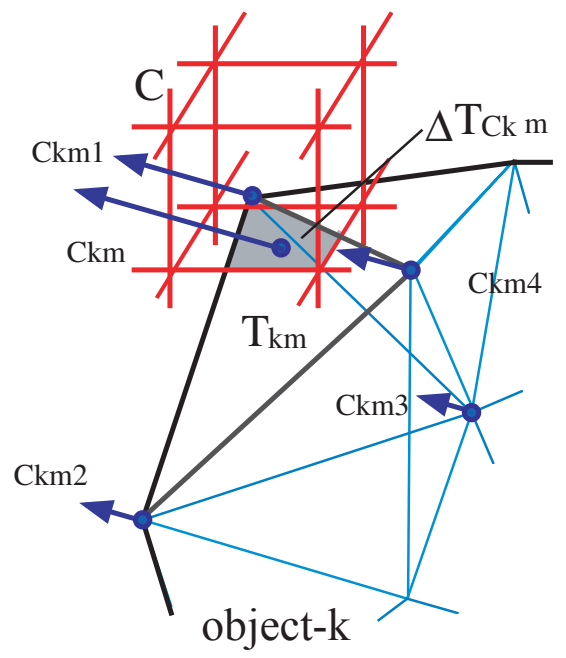

Figure 1: Estimation of the fluid force acting on an object.

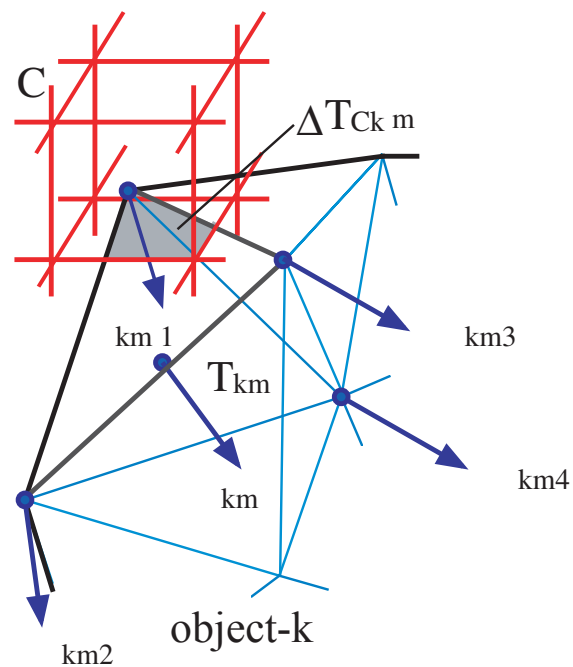

Figure 2: Transformation from object motion to velocity in multiphase field.

vector $\boldsymbol{u}$ in the multiphase field, which is defined at the center of the fluid-cell, is determined as the following mass-averaged value:

$$
\boldsymbol{u}=\frac{1}{m_{C}}\left(m_{f} \boldsymbol{u}_{f}+\sum_{k} \sum_{m} \rho_{b k} \Delta T_{C k m} \boldsymbol{v}_{k m}\right)
$$




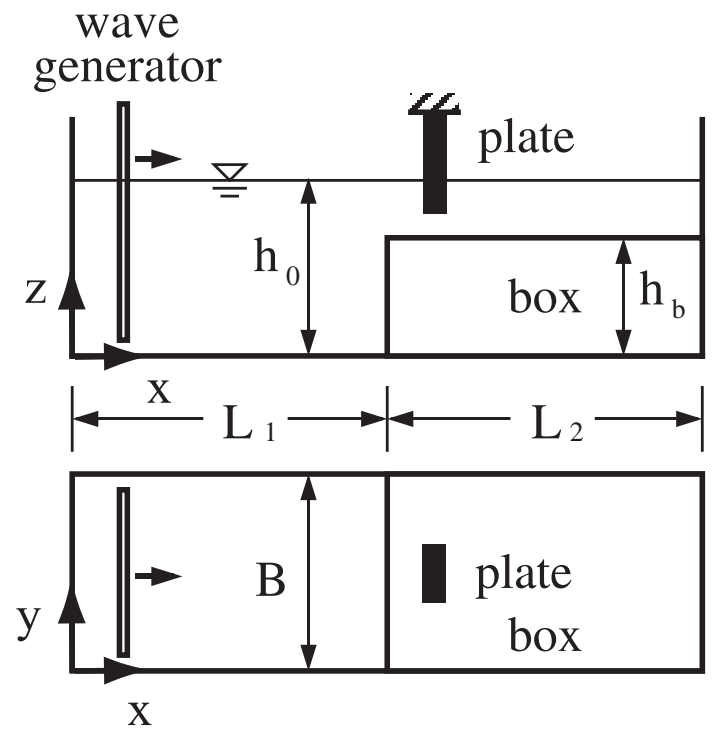

Figure 3: Water tank used in experiments (side and plane views).

where $m_{C}$ and $m_{f}$ are total mass in the fluid-cell and the mass of gas and liquid phases, respectively. The velocity vector of the mixture of gas and liquid phases is given by $\boldsymbol{u}_{f}$.

\section{Application to experimental results}

\subsection{Experiments}

As shown in Fig. 3, the deformations of an elastic plate were measured in a water tank equipped with a wave generator. The wave-induced flow is generated on a box in the water tank shown in Fig. 3, which causes the deformation of the plate. The top of the elastic plate is fixed on a steel plate, on which four strain gages are attached to measure the fluid forces acting on the elastic plate. The lengths of the tank $L_{1}, L_{2}$ and $B$ shown in Fig. 3 are $0.7 \mathrm{~m}, 0.7 \mathrm{~m}$ and $0.19^{\circ} \mathrm{m}$, while the initial water depth $h_{0}$ and the height of the box $h_{b}$ are $0.15 \mathrm{~m}$ and $0.1 \mathrm{~m}$. The bottom surface of the elastic plate is placed $15 \mathrm{~mm}$ above the top of the box. The elastic plate, $10 \times 30 \times 90 \mathrm{~mm}$, is made of a soft rubber and its density is about 0.255 . The linearity of the constitutive relation was experimentally confirmed and its Young's modulus and dumping coefficient per volume are $3.5 \times 10^{5} \mathrm{~Pa}$ and $2.0 \times 10^{3} \mathrm{~N} \mathrm{~s} / \mathrm{m} / \mathrm{m}^{3}$.

In the experiments, two cases of waves were generated; the maximum water depths $h_{m}$ in front of the box are $185 \mathrm{~mm}$ in case-A and $195 \mathrm{~mm}$ in case-B. In 


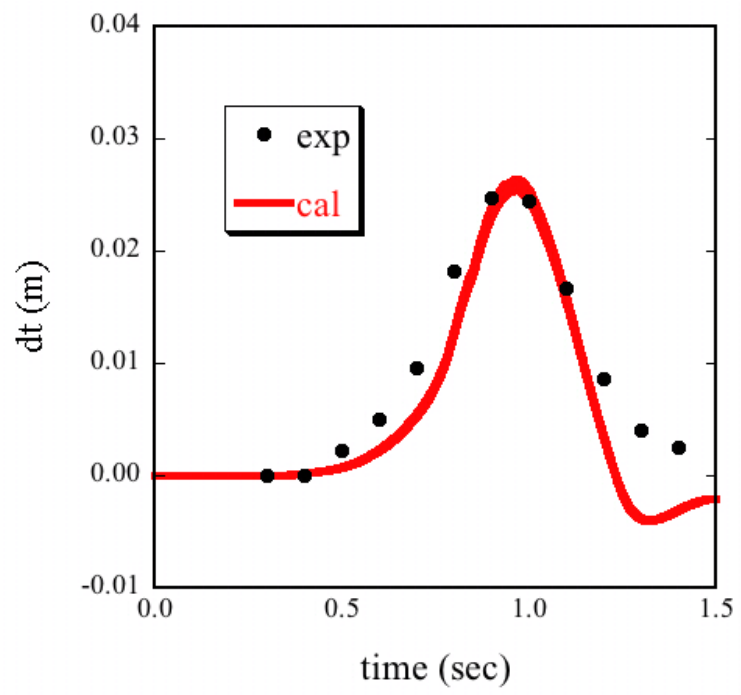

(a) case- $\mathrm{A}$

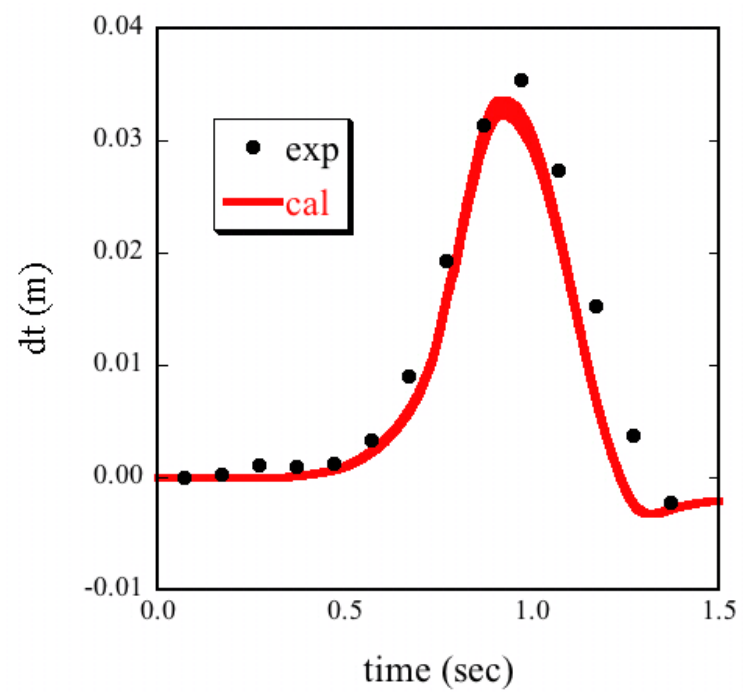

(b) case-B

Figure 4: Displacements of bottom end of elastic plate.

both cases, the deformations of the elastic plate were recorded by a video camera and the displacements were evaluated with the image analysis. In addition, the fluid forces were measured by the strain gages and the natural oscillations of the plate were removed from the output signals by applying a moving-average filter. 
42 Fluid Structure Interaction V

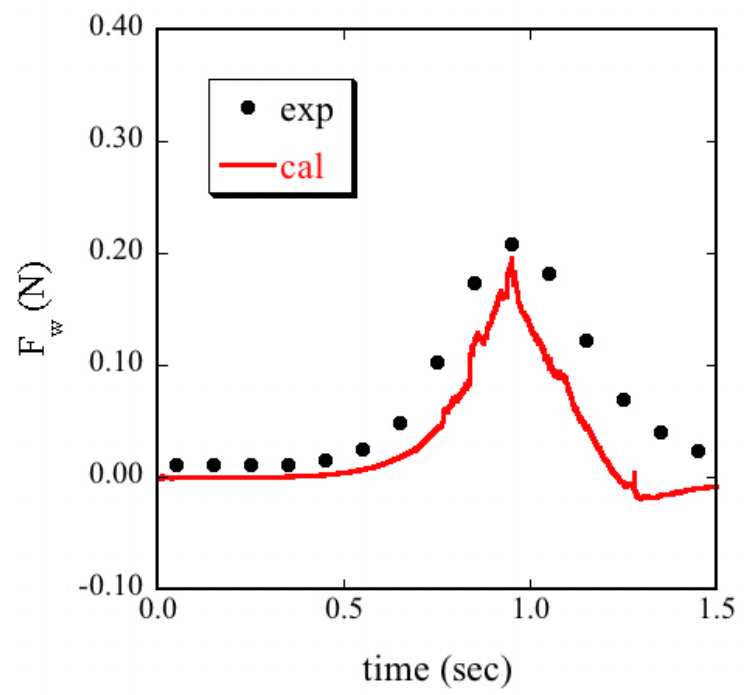

(a) case- $\mathrm{A}$

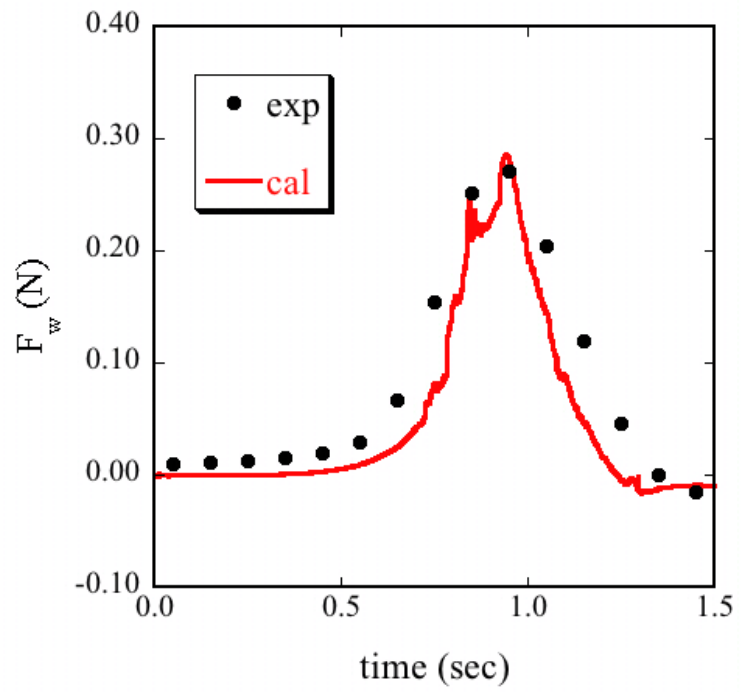

(b) case-B

Figure 5: Fluid forces acting on elastic plate.

\subsection{Computational results and discussion}

The computational method was applied to the experimental results to confirm its validity. In the computations, a fluid-cell is a $10 \times 10 \times 10 \mathrm{~mm}$ cube and $140 \times$ 


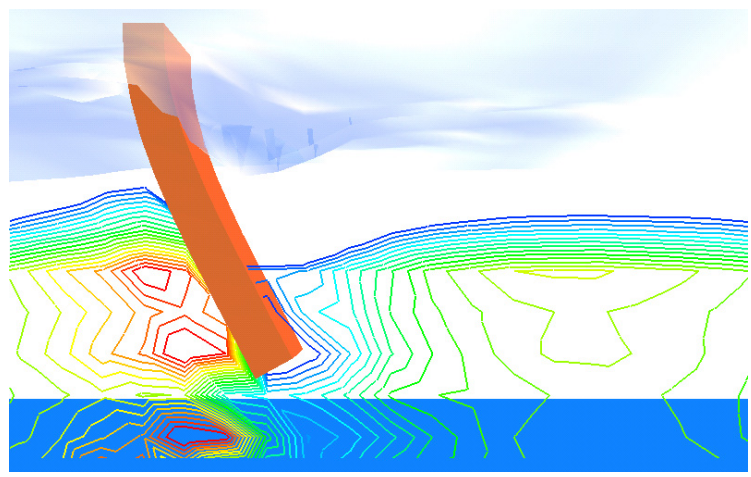

(a) side view

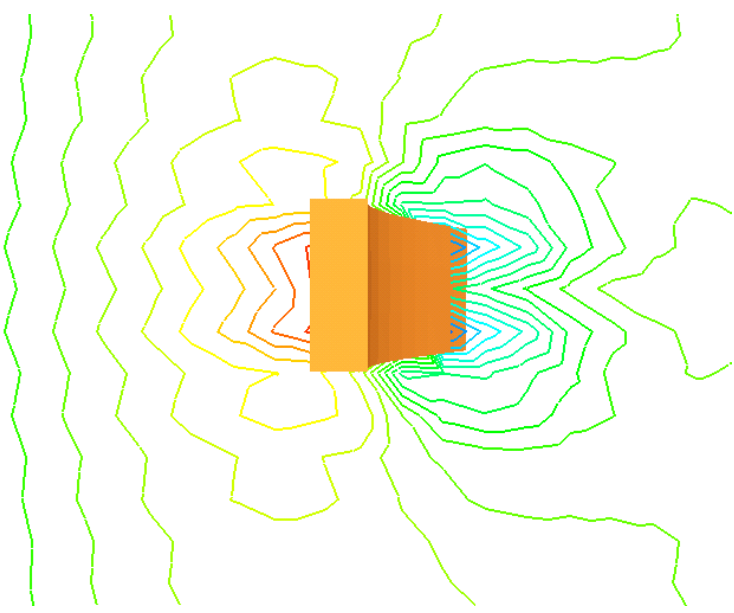

(b) top view

Figure 6: Predicted pressure distributions around deformed plate (case-B).

$19 \times 25$ cells were set for the regions inside of the tank, including water, air and the elastic plate. The kinematic viscosities of water and air are set at $1.0 \times 10^{-6}$ and $1.0 \times 10^{-5} \mathrm{~m}^{2} / \mathrm{s}$, while their densities are $1.0 \times 10^{3}$ and $1.0 \mathrm{~kg} / \mathrm{m}^{3}$ respectively. The elastic plate is represented by 164 tetrahedron elements with 441 nodes. The experimentally obtained Young's modulus and damping coefficient are used in the computation.

Figure 4 shows the time histories of the displacements $d t$ at the bottom of the elastic plate. The displacements of case-B are larger than those of case-A due to the difference of the wave heights. Since the predicted results generally agree with the experimentally-observed displacements in both cases, it can be thought that the present solid model for finite deformation is effective.

The comparisons between experiments and predictions regarding the fluid forces $F_{w}$ acting on the plate are shown in Fig. 5. While the calculated fluid forces are 


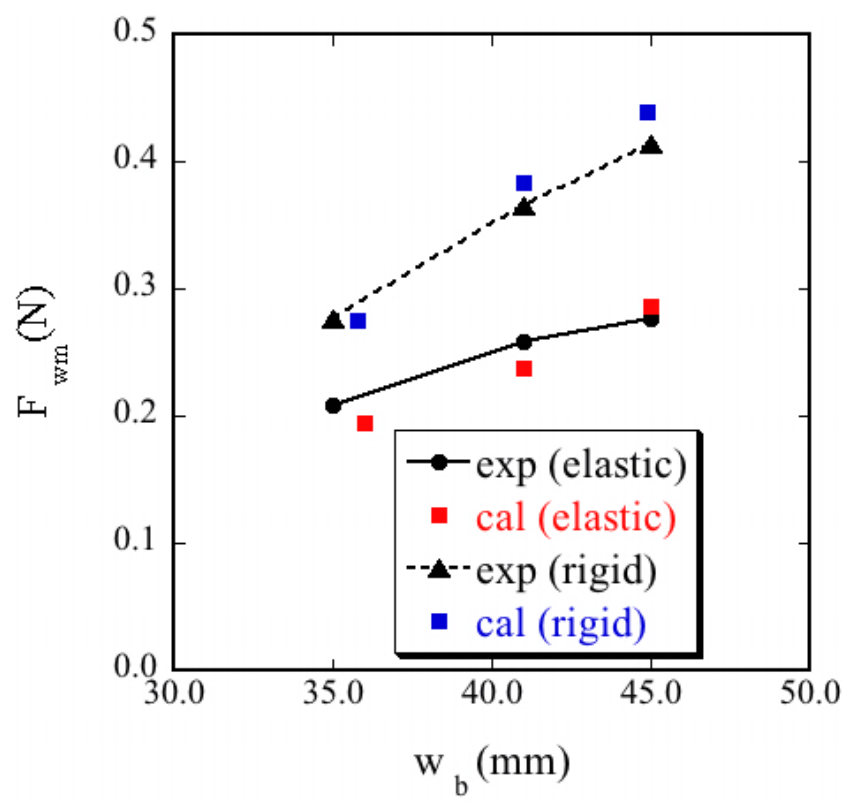

Figure 7: Fluid forces acting on elastic and rigid plates.

slightly smaller than those obtained in experiments, the shapes of the distributions are reasonably predicted. The pressure distributions around the deformed plate are shown in Fig. 6. It can be seen that the high pressure regions exist on the upstream ( $-x$ direction) side of the wave-induced flow, while on the downstream side low pressure zone arises due to the wake vortex flows. It can be concluded that the adequate evaluation of the surrounding pressure field enables us to obtain the reasonably-predicted results as shown in Fig. 4 and Fig. 5.

Finally, Fig. 7 shows the peak values of the fluid forces $F_{w m}$ acting on elastic and rigid plates, which have the same geometries. While both of the fluid forces increase with increasing wave height $w_{b}\left(=h_{m}-h_{0}\right)$, the forces against the elastic plate are smaller than those acting on the rigid one. This is due to the fact that a part of the fluid forces are consumed in the work to deform the object in case that the plate is elastic. Including these tendencies, the experimentally-observed $F_{w m}$ are adequately predicted with the present computational method.

\section{Conclusions}

On the basis of the multiphase modeling, the computational method has been proposed to predict the interactions between free-surface flows and the linear elastic objects which undergo finite deformations. In the multiphase modeling, the gas, liquid and solid phases are treated as immiscible and incompressible fluids with different physical properties and the governing equations are derived with the one- 
fluid modeling. The solid model, on the other hand, is derived with objective stress rates to deal with the finite deformations.

The proposed computational method was applied to the experimental results; deformations of an elastic plate due to the wave-induced flows and the fluid forces acting on it. As a result, it was shown that the time histories of the plate displacements and fluid forces are successfully predicted with the present method. In addition, the maximum values of the fluid forces $F_{w m}$ acting on the elastic and rigid plates were compared and it was demonstrated that the relationships between wave heights and $F_{w m}$ are reasonably predicted.

\section{References}

[1] Xiao, F., Yabe, T., Ito, T. \& Tajima, M., An algorithm for simulating solid objects suspended in stratified flow, Computer Physics Communications, Vol. 102, pp. 147-160, 1997.

[2] Ushijima, S., Yamada, S., Fujioka, S. \& Nezu, I., Prediction method (3D MICS) for transportation of solid bodies in 3D free-surface flows, JSCE Journal, Vol. 810/II-74, pp. 79-89, 2006.

[3] Zang, Y., Street, R. L. \& Koseff, J. R., A non-staggered grid, fractional step method for time-dependent incompressible Navier-Stokes equation in curvilinear coordinates, Journal of computational physics, Vol. 114, pp. 18-33, 1994.

[4] Harlow, F. H. \& Welch, J. E., Numerical calculation of time-dependent viscous incompressible flow of fluid with free surface, Phys. Fluids, Vol. 8, No. 12, pp. 2182-2189, 1965.

[5] Ushijima, S. \& Nezu, I., Higher-order implicit (C-ISMAC) method for incompressible flows with collocated grid system, JSCE Journal, No. 719/II-61, pp. 21-30, 2002.

[6] Ushijima, S. \& Okuyama, Y., Comparison of C-HSMAC and SOLA methods for pressure computation of incompressible fluids, JSCE Journal, No. 747/II65, pp. 197-202, 2003.

[7] Ushijima, S., Multiphase-model approach to predict arbitrarily-shaped, Proc. APCOM'07, 2007.

[8] Bonet, J. \& Wood, R. D., Nonlinear Continuum Mechanics for Finite Element Analysis, Cambridge Univ. Press, 2008.

[9] Doig, R., Okazawa, S. \& Fujikubo, M., Solid-fluid interaction analysis by using amulti-material Eulerian finite element method, Journal of Applied Mechanics JSCE, Vol. 9, pp. 151-159, 2006. 